\title{
Aborder le raisonnement clinique du point de vue pédagogique
}

\section{Les difficultés de raisonnement clinique à l'étape du recueil initial des données et de la génération d'hypothèses}

\section{Tackling clinical reasoning from a pedagogical perspective \\ II. Clinical reasoning difficulties at the hypothesis generation and data gathering stage}

\section{Marie-Claude AUDÉTAT, Suzanne LAURIN et Gilbert SANCHE}

Département de médecine familiale et de médecine d'urgence, CPASS (Centre de pédagogie appliquée aux sciences de la santé), Université de Montréal, Montréal, Canada

Manuscrit reçu le 6 juillet 2011 ; commentaires éditoriaux formulés aux auteurs le 30 août et le 7 novembre 2011 ; accepté pour publication le 14 novembre 2011

\section{Mots-clés \\ Raisonnement clinique ; supervision clinique ; diagnostic pédagogique \\ Keywords Clinical reasoning; clinical supervision; educational diagnosis}

Messages clés - Lors du raisonnement clinique, un recueil efficace des données s'articule autour de la sélection et de l'interprétation des éléments-clés d'une situation clinique qui permettent la génération et la vérification stratégique d'hypothèses diagnostiques.

En appliquant une démarche de diagnostic pédagogique, similaire à celle appliquée lors du raisonnement clinique, le clinicien enseignant peut identifier, en supervision clinique, les difficultés de raisonnement clinique qui se manifestent à cette étape et tenter de les corriger.

Key messages - During clinical reasoning, efficient data gathering is based on selecting and interpreting the key elements of a clinical situation, which helps strategically generate and verify diagnostic hypotheses.

Through an educational diagnosis approach similar to the one used in clinical settings, clinician teachers can, in clinical supervision, identify clinical reasoning difficulties that arise at this stage and try to correct them.
Cet article est le deuxième d'une série didactique de six chroniques qui s'appuient sur des travaux de recherche ayant permis d'élaborer une taxonomie des difficultés de raisonnement clinique, de décrire un processus de diagnostic pédagogique et de distinguer des stratégies de remédiation spécifiques ${ }^{[1]}$. Le premier article présentait le cadre conceptuel du diagnostic pédagogique des difficultés de raisonnement 
clinique, telles qu'elles se manifestent quotidiennement dans le contexte d'apprentissage en milieu clinique $^{[2]}$.

Cette deuxième chronique aborde les difficultés de raisonnement clinique qui concernent l'étape du recueil initial des données et de la génération d'hypothèses, telles qu'elles peuvent se présenter dans le contexte d'une supervision différée (indirecte).

Ce type de difficulté peut faire partie de la progression normale d'un apprenant et son identification rapide peut permettre aux superviseurs de soutenir l'évolution du raisonnement vers le niveau attendu, en évitant ainsi que des difficultés éventuelles ne se cristallisent et se transforment en impasses pédagogiques. Rappelons que plusieurs difficultés de raisonnement clinique peuvent coexister ou être associées à des lacunes d'autres ordres (affectifs, relationnels ou $\operatorname{organisationnels}^{[3,4]}$.

\section{Exemple clinique}

Une femme de 55 ans, hypertendue et asthmatique, consulte pour un problème respiratoire apparu depuis un mois.

Après sa consultation, l'apprenant (un externe ou un interne/résident, selon le cas) vous présente le cas longuement, en énumérant tous les détails et en utilisant les propres mots de la patiente. Vous constatez qu'il a oublié les réponses de sa patiente à certaines questions déterminantes, qui auraient pu lui permettre d'évoquer une hypothèse alternative ou d'en exclure une autre. Il a aussi omis certains éléments de l'examen physique qui vous paraissent importants.

Enfin, vous avez l'impression - un peu irritante de « devoir faire tout le travail » d'organisation et de pondération des éléments recueillis lors de cette entrevue pour vous en faire une idée claire.

Vous avez aussi remarqué, au cours des dernières supervisions de cet apprenant, que celui-ci, après avoir rencontré ses patients, avait besoin de prendre beaucoup de temps pour consulter ses notes avant de pouvoir vous en parler.

Cette fois, vous êtes allé à sa rencontre dès qu'il est sorti du cabinet de consultation et avez décidé d'essayer de mieux comprendre sa démarche.
- Superviseur (après avoir écouté le long récit) : J'aimerais que tu résumes la situation clinique en quelques phrases.

- Apprenant (regardant ses notes) : C'est une dame de 55 ans qui a du mal à respirer quand elle monte les escaliers chez elle, surtout quand elle porte des paquets.....

- Superviseur : J'aimerais que tu utilises des termes médicaux pour faire une synthèse et que tu le fasses sans regarder tes notes. Par exemple, une femme de 55 ans qui présente une dyspnée d'effort...

- Apprenant: Vous me laissez quelques minutes pour y penser? D'habitude, je prends un moment pour réfléchir à ce que j’ai trouvé pendant la consultation avant d'en parler au superviseur.

\section{Les problèmes liés à la génération $d$ 'hypothèses diagnostiques}

\section{Que désigne-t-on par " difficulté à générer des hypothèses diagnostiques " ?}

Cette difficulté survient lorsque le clinicien n'identifie pas, ne sélectionne pas ou n'interprète pas adéquatement des éléments-clés ou des indices qui pourraient l'orienter et susciter la génération de quelques hypothèses diagnostiques dès le début de l'entrevue. En conséquence, son raisonnement clinique ne s'articule pas, ni ne s'oriente de façon à pouvoir préciser et cibler son recueil de données pour vérifier ces hypothèses. L'anamnèse ne tient donc pas compte de ces indices et reste dès lors non stratégique, c'est-à-dire non orientée par l'examen critique des hypothèses.

\section{Comment reconnaître une difficulté à générer des hypothèses diagnostiques?}

Le tableau I présente quelques exemples d'indices qui auraient pu être présents et auraient permis de déceler le problème de génération d'hypothèses. Les éléments 
relatifs à l'exemple formulé ci-dessus et identifiables en supervision différée (indirecte) se retrouvent dans les cases tramées du tableau.

\section{Comment expliquer la difficulté à générer des hypothèses diagnostiques ?}

Après avoir identifié des manifestations de problèmes dans la génération d'hypothèses, le rôle du superviseur est d'utiliser son raisonnement pédagogique pour chercher à en expliquer les raisons afin de les corriger. Il aidera d'abord l'apprenant à prendre conscience du problème pour tenter d'en comprendre les causes.

- Superviseur : J'ai remarqué que tu prenais beaucoup de temps entre les rencontres cliniques et le moment de présenter le cas... c'est pour réviser tes notes?

- Apprenant : J'aime bien prendre un moment pour me faire une synthèse des éléments recueillis et me faire une idée des diagnostics possibles.

- Superviseur : Effectivement, cela peut être utile, mais tu n'auras pas ce temps plus tard! Par ailleurs, il est important que tu te fasses une idée au fur et à mesure que tu poses tes questions et que tu construis ton raisonnement. Dès les premières minutes de la consultation, quelques premières hypothèses devraient te venir en tête et guider ton anamnèse. Regardons ensemble dans cette situation ce que cela pourrait donner (...)

- Superviseur : Tu as tendance aussi à me raconter tout ce que la patiente t'a dit, tout ce que tu as pu observer et parfois tu oublies des éléments importants que t'a fournis la patiente. Sais-tu pourquoi cela se passe ainsi?

- Apprenant : Il y a beaucoup d'informations à retenir! J'ai peur d'en oublier. La dyspnée est un symptôme qui ouvre vers de nombreux diagnostics différentiels... je préfère les vérifier systématiquement et faire la synthèse ensuite.

Le tableau II illustre plusieurs hypothèses qui peuvent expliquer la difficulté de l'apprenant à générer des hypothèses. Il est pertinent de les distinguer, car les correctifs pourront varier en fonction des causes.

\section{Comment corriger la difficulté à générer des hypothèses diagnostiques?}

Une fois que le problème pédagogique est mieux défini, la dernière étape du raisonnement pédagogique consiste à mettre en place un plan d'intervention qui comprendra différentes stratégies, adaptées aux objectifs préalablement circonscrits. Quelques exemples sont présentés dans le tableau III.

Ces interventions peuvent se faire lors de la supervision ou après, lorsque l'apprenant et l'enseignant sont plus libres de leur temps.

\section{Conclusion}

Les difficultés qu'éprouvent les étudiants - externes ou internes/résidents - à l'étape du recueil initial des données et de la génération d'hypothèses ne sont pas toujours faciles à détecter en supervision différée (indirecte). Le superviseur doit être attentif au sentiment qu'il perçoit de ne pas être capable de suivre le déroulement $\mathrm{du}$ raisonnement tel que le rapporte l'étudiant, cette forme d' « intuition » pouvant constituer un indice de difficultés potentielles.

L'observation des consultations peut permettre de documenter et de vérifier les hypothèses pédagogiques générées à partir de ces indices. Ainsi, l'anamnèse effectuée sous forme de questionnaire systématique, exhaustif, abordant les systèmes ou appareils de façon cloisonnée (selon une approche dite « en silo »), dans un ordre qui ne tient pas compte des caractéristiques de la situation clinique, est un indice fréquent d'une difficulté à générer des hypothèses. Certains de ces indices ne peuvent être mis en évidence qu'en supervision directe.

Cette façon de mener une entrevue clinique, quoique normale pour un apprenant peu expérimenté, doit évoluer vers une structure plus souple qui favorise un raisonnement clinique efficace. Le superviseur, par son soutien pédagogique, peut y contribuer significativement. Le guide d'aide au diagnostic pédagogique et au développement de stratégies de remédiation a pour objectif d'aider le clinicien enseignant dans sa tâche pédagogique. Il est disponible en ligne en tant que matériel éditorial complémentaire. 


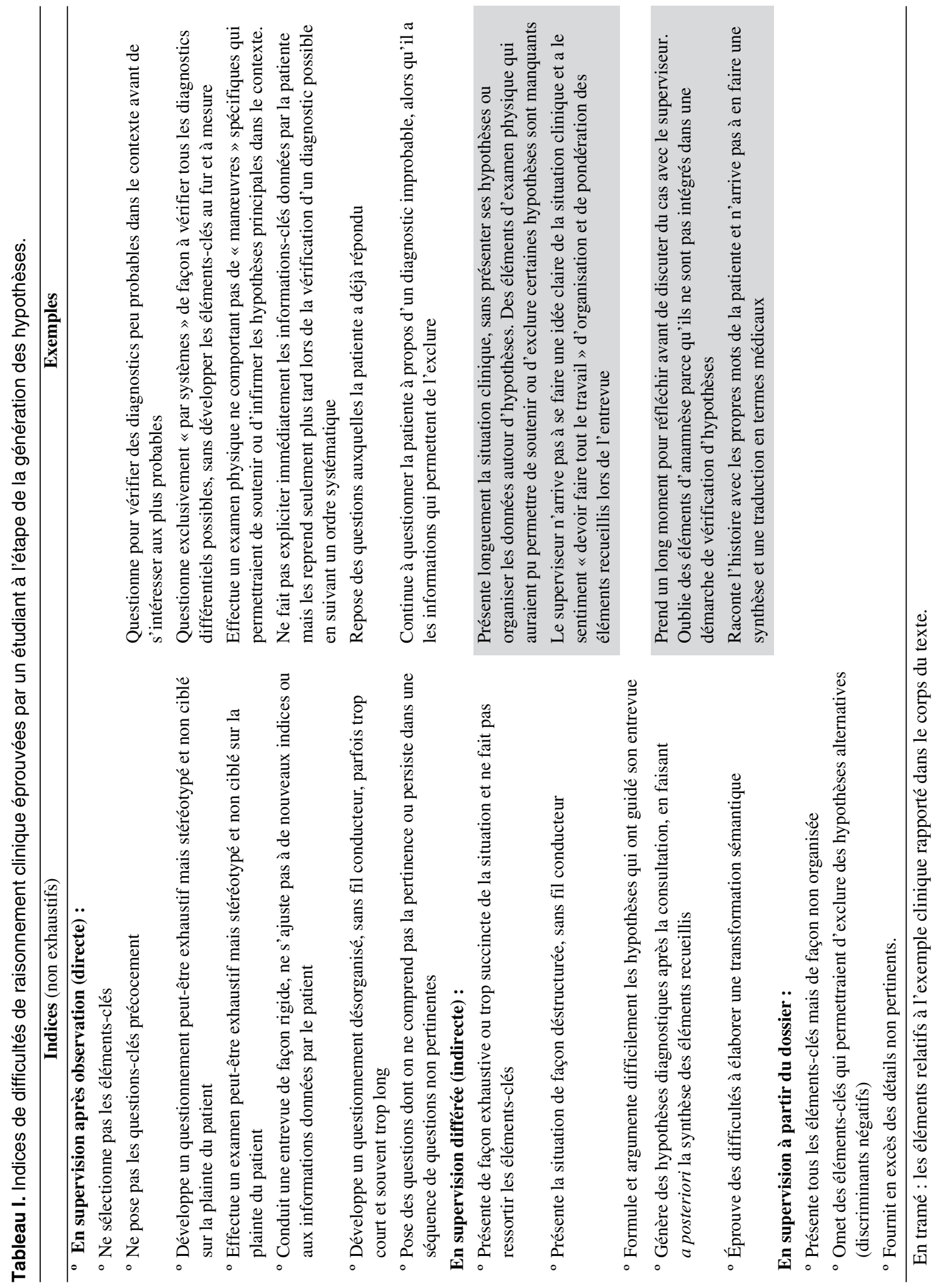


Tableau II. Hypothèses pédagogiques permettant d'expliquer la difficulté à générer des hypothèses diagnostiques.

\section{Hypothèses explicatives de la difficulté à générer des Exemples hypothèses (non exhaustives)}

- Déficit de connaissances ou d'expérience avec ce type de cas

- Difficulté à effectuer une transformation sémantique

L'apprenant a des difficultés à se faire une représentation du problème et à transformer l'information obtenue en « concept médical ». Par exemple : traduire « un essoufflement dans les escaliers » en « dyspnée à l'effort modéré »

- Inexpérience ou insécurité, besoin de poser les questions dans un ordre établi pour se sécuriser, pour éviter de perdre le fil

Préoccupé par la crainte d'oublier de vérifier un diagnostic possible, le résident aborde les systèmes «en silo » (de façon cloisonnée) sans suivre les indices donnés par la patiente

Ne sait pas qu'il peut se permettre de questionner en passant d'un système à l'autre, de façon à vérifier des hypothèses au fur et à mesure qu'elles se présentent à son esprit

En tramé : les éléments relatifs à l'exemple clinique rapporté dans le corps du texte.

Tableau III. Stratégies de correction des difficultés de génération d'hypothèses.

\begin{tabular}{|c|c|}
\hline Objectifs & Actions \\
\hline $\begin{array}{l}\text { Expliciter le processus de raisonnement } \\
\text { clinique en général }\end{array}$ & $\begin{array}{l}\text { - Explicitation pas à pas des différentes étapes du raisonnement. } \\
\text { "Reprenons ensemble les étapes du raisonnement clinique dans cette } \\
\text { situation » }\end{array}$ \\
\hline \multirow{2}{*}{$\begin{array}{l}\text { Verbaliser et expliciter sa propre } \\
\text { représentation du problème et son propre } \\
\text { raisonnement clinique }\end{array}$} & $\begin{array}{l}\text { - Rendre visible et accessible le processus de génération précoce } \\
\text { d'hypothèses : «Tu vois, quandj'entends « difficulté à respirer » chez une } \\
\text { dame hypertendue et asthmatique de } 55 \text { ans, je pense à insuffisance } \\
\text { cardiaque et asthme et, après avoir fait préciser la plainte principale, je } \\
\text { vais poser des questions pour vérifier d'abord ces deux hypothèses }\end{array}$ \\
\hline & $\begin{array}{l}\text { - Expliciter et illustrer l'étape de la transformation sémantique: «quand ta } \\
\text { patiente dit qu'elle est essoufflée quand elle monte les escaliers, mais pas } \\
\text { en marchant sur un terrain plat, je pense : dyspnée à l'effort modéré; } \\
\text { dans le cas que tu me décris, j'ajouterais : accompagnée de douleurs } \\
\text { thoraciques soulagées par le repos» }\end{array}$ \\
\hline $\begin{array}{l}\text { Développer la génération systématique } \\
\text { d'hypothèses précoces }\end{array}$ & $\begin{array}{l}\text { Dans le cas de cette patiente, quelles hypothèses ont guidé ton anamnèse? } \\
\text { Lors de notre prochaine matinée de supervision clinique, j'aimerais qu'on } \\
\text { se fixe comme objectif d'être attentif à générer deux hypothèses à vérifier } \\
\text { dès le début de la consultation }\end{array}$ \\
\hline $\begin{array}{l}\text { Développer la reconnaissance d'indices } \\
\text { discriminants en début d'entrevue }\end{array}$ & $\begin{array}{l}\text { - Nous allons enregistrer ta prochaine consultation et nous en regarderons } \\
\text { quelques minutes en identifiant ensemble les indices que tu aurais pu } \\
\text { approfondir et les hypothèses diagnostiques que tu aurais pu générer et } \\
\text { vérifier }\end{array}$ \\
\hline
\end{tabular}

En tramé : les éléments relatifs à l'exemple clinique rapporté dans le corps du texte. 


\section{Contributions}

Les trois auteurs ont conjointement mené des discussions sur le contenu et la structuration de l'article. Marie-Claude Audétat et Suzanne Laurin ont rédigé une première version qui a fait l'objet d'une révision par Gilbert Sanche. La version finale du manuscrit a été approuvée par les trois auteurs.

\section{Références}

1. Audétat MC, Laurin S, Sanche G, Béïque C, Caire-Fon $\mathrm{N}$, Blais J et al. Clinical reasoning difficulties: a taxonomy for clinical teachers. Med Teach 2011. Soumis à publication.
2. Audétat M, Laurin S, Sanche G. Aborder le raisonnement clinique du point de vue pédagogique. Un cadre conceptuel pour identifier les problèmes de raisonnement clinique chez les étudiants. Pédagogie Médicale 2011;12:223-30.

3. Vaughn LM, Baker RC, De Witt TG. The problem learner. Teach Learn Med 1998;10:217-22.

4. Steinert Y, Lewitt C. Working with the «problem» resident; guidelines for definition and intervention. Fam Med 1993;25:627-32.

Correspondance et offprints : Marie-Claude Audétat, Centre de pédagogie appliquée aux sciences de la santé (CPASS), Faculté de médecine, Université de Montréal, CP 6128, succ. Centre Ville, Montréal (QC) H3C 3J7, Canada. Mailto :

mcaudetat@sympatico.ca 